\title{
A KHINCHIN SEQUENCE
}

\author{
THOMAS WIETING
}

(Communicated by Jonathan M. Borwein)

Abstract. We prove that the geometric and harmonic means of the sequence $Z_{2}$ of positive integers proposed by Bailey, Borwein, and Crandall converge to the corresponding Khinchin Constants.

\section{Khinchin Sequences}

One defines the Khinchin Constant $K$ by the following relation:

$$
\log (K)=\frac{1}{\log (2)} \sum_{k=1}^{\infty} \log (k) \log \left(\frac{(k+1)^{2}}{k(k+2)}\right)=\log (2.685452001 \cdots) .
$$

For any sequence $A=\left(a_{j}\right)$,

$$
A: \quad a_{1}, a_{2}, a_{3}, \ldots, a_{j}, \ldots
$$

of positive integers, let us refer to $A$ as a Khinchin Sequence iff the geometric means of $A$ converge to $K$ :

$$
\lim _{n \rightarrow \infty}\left(\prod_{j}^{n} a_{j}\right)^{1 / n}=K
$$

That is,

$$
\lim _{n \rightarrow \infty} \frac{1}{n} \sum_{j=1}^{n} \log \left(a_{j}\right)=\log (K) .
$$

For any irrational number $x$ in the interval $(0,1)$, let us refer to $x$ as a Khinchin Number iff the continued fraction expansion $A(x)=\left(a_{j}(x)\right)$

$$
A(x): \quad a_{1}(x), a_{2}(x), a_{3}(x), \ldots, a_{j}(x), \ldots
$$

for $x$ is a Khinchin Sequence. In this paper, our objective is to prove that the particular sequence $C=\left(c_{j}\right)$

$$
C: \quad 2,5,1,11,1,3,1,22,2,4,1,7,1,2,1,45,2,4,1,8, \ldots, c_{j}, \ldots
$$

of positive integers proposed by Bailey, Borwein, and Crandall is a Khinchin Sequence. See reference [1].

Received by the editors January 12, 2007.

2000 Mathematics Subject Classification. Primary 11Y65; Secondary 28D05.

Key words and phrases. Khinchin Sequence, continued fraction expansion, geometric mean, harmonic mean.

Thanks to R. C. Crandall for suggesting the subject of this paper. 
In the paper just cited, the authors denote $C$ by $Z_{2}$. They define the sequence $C$ in terms of two auxiliary sequences $U=\left(u_{j}\right)$ and $V=\left(v_{k}\right)$, defined in turn as follows. The first sequence, $U$, is the van der Corput Sequence:

$$
U: \quad \frac{1}{2}, \frac{1}{4}, \frac{3}{4}, \frac{1}{8}, \frac{5}{8}, \frac{3}{8}, \frac{7}{8}, \frac{1}{16}, \frac{9}{16}, \frac{5}{16}, \frac{13}{16}, \frac{3}{16}, \frac{11}{16}, \frac{7}{16}, \frac{15}{16}, \ldots, u_{j}, \ldots
$$

Specifically, for each positive integer $j, u_{j}$ is the dyadic rational number obtained by reflecting the binary representation of $j$ in the binary point. For example, $u_{12}:=0.0011=3 / 16$ because $12=1100.0$. See reference [2]. The second sequence, $V$, describes a particular partition of the interval $(0,1]$ :

$$
V: \quad \cdots<v_{k}=\frac{1}{\log (2)} \log \left(\frac{k+1}{k}\right)<\cdots<v_{3}<v_{2}<v_{1}=1 .
$$

Now, in terms of $U$ and $V$, Bailey, Borwein, and Crandall define the sequence $C$ as follows:

$$
C: \quad c_{j}=k \Longleftrightarrow v_{k+1}<u_{j} \leq v_{k} .
$$

For example, $c_{12}=7$ because $v_{8}<u_{12} \leq v_{7}$.

\section{Motivation}

To set a context for our study of the sequence $C$, let us describe a special case of the Ergodic Theorem. Let $\lambda$ stand for Lebesgue Measure, defined as usual on $\mathbb{R}$. Let $X$ be the set of all irrational numbers in the interval $(0,1)$. Let $\mu$ stand for Gauss Measure, defined on $X$ as follows:

$$
\mu(E):=\frac{1}{\log (2)} \int_{E} \frac{1}{1+x} \lambda(d x)
$$

where $E$ is any Borel subset of $X$. Note that $\mu(E)=0$ iff $\lambda(E)=0$. Let $F$ be the mapping carrying $X$ to itself defined as follows:

$$
F(x):=\frac{1}{x}-\left\lfloor\frac{1}{x}\right\rfloor
$$

where $x$ is any number in $X$. Of course, $\lfloor 1 / x\rfloor$ denotes the largest among all integers $\ell$ for which $\ell \leq 1 / x$. Note that $F$ is continuous. One may view the ordered pair $(X, F)$ as a (discrete) dynamical system. For any $x$ in $X$, one may say that if the system is in state $x$ at time 0 , then the system is in state $F(x)$ one unit of time later. By an elementary argument, one can show that $\mu$ is invariant under $F$, in the sense that, for any Borel subset $E$ of $X, \mu\left(F^{-1}(E)\right)=\mu(E)$. By a more substantial argument, one can also show that $\mu$ is ergodic under $F$, in the sense that, for any Borel subset $E$ of $X$, if $F^{-1}(E)=E$, then either $\mu(E)=0$ or $\mu(E)=1$. See reference [4]. Let $h$ be the function defined on $X$ as follows:

$$
h(x):=\left\lfloor\frac{1}{x}\right\rfloor
$$

where $x$ is any number in $X$. Note that $h$ is continuous and that the values of $h$ are positive integers. One may refer to $h$ as an observable for the given dynamical system.

For any $x$ in $X$, one obtains the orbit $O(x)=\left(x_{j}\right)$ of $x$ under $F$,

$$
O(x): \quad x=x_{1}, x_{2}, x_{3}, \ldots
$$


and one obtains the corresponding (discrete) time sequence $A(x)=\left(a_{j}(x)\right)$,

$$
A(x): \quad a_{1}(x), a_{2}(x), a_{3}(x), \ldots,
$$

where

$$
x_{j}:=F^{j-1}(x), \quad a_{j}(x):=h\left(x_{j}\right) .
$$

The sequence $A(x)$ is the Continued Fraction Expansion for $x$.

For the assembly $X, \mu, F$, and $\log (h)$, the Ergodic Theorem states that, for almost every $x$ in $X$,

$$
\lim _{n \rightarrow \infty} \frac{1}{n} \sum_{j=1}^{n} \log \left(h\left(F^{j-1}(x)\right)\right)=\int_{X} \log (h(y)) \mu(d y) .
$$

That is, the time average of $\log (h)$ over $O(x)$ equals the space average of $\log (h)$ over $X$. See reference [5]. Hence,

$$
\begin{aligned}
\lim _{n \rightarrow \infty} \frac{1}{n} \sum_{j=1}^{n} \log \left(a_{j}(x)\right) & =\int_{X} \log (h(y)) \mu(d y) \\
& =\sum_{k=1}^{\infty} \log (k) \mu\left(\frac{1}{k+1}, \frac{1}{k}\right) \\
& =\frac{1}{\log (2)} \sum_{k=1}^{\infty} \log (k) \log \left(\frac{(k+1)^{2}}{k(k+2)}\right) \\
& =\log (K) .
\end{aligned}
$$

Now it is plain that, for almost every irrational number $x$ in the interval $(0,1), x$ is a Khinchin Number. However, no particular examples of such numbers are known. The beguiling cases of $\pi-3$ and even of $K-2$ itself have been studied energetically but to no analytic decision as yet. In reference [3], one may find the optimistic opinion that $\pi-3$ is a Khinchin Number. The graphs displayed in Figures 1 and 2 suggest a more cautious, though still hopeful, opinion on $\pi-3$ and on $K-2$ as well. The graphs are list plots of the following data:

$$
\frac{1}{n} \sum_{j=1}^{n} \log \left(a_{j}(x)\right)-\log (K) \quad(1 \leq n \leq 4096)
$$

where $x=\pi-3$ and $x=K-2$.

Failing to identify particular Khinchin Numbers, one naturally turns to the design of particular Khinchin Sequences. One might, for instance, design sequences $A=\left(a_{j}\right)$ such that, for each $j, a_{j}$ equals 2 or 3 and such that the $2 \mathrm{~s}$ and $3 \mathrm{~s}$ occur in $A$ in correct "limiting proportions," specifically, the proportions $p$ and $q$, where $p$ and $q$ are the positive numbers for which $p+q=1$ and $\log (K)=p \log (2)+q \log (3)$. However, such a design would be very difficult to implement, since it depends upon the calculation of $\log (K)$ to arbitrary accuracy. In sharp contrast, Bailey, Borwein, and Crandall have proposed a particular candidate for a Khinchin Sequence, namely, the sequence $C$, which they have defined in constructive and rapidly computable terms. Let us prove formally that $C$ is indeed a Khinchin Sequence. 


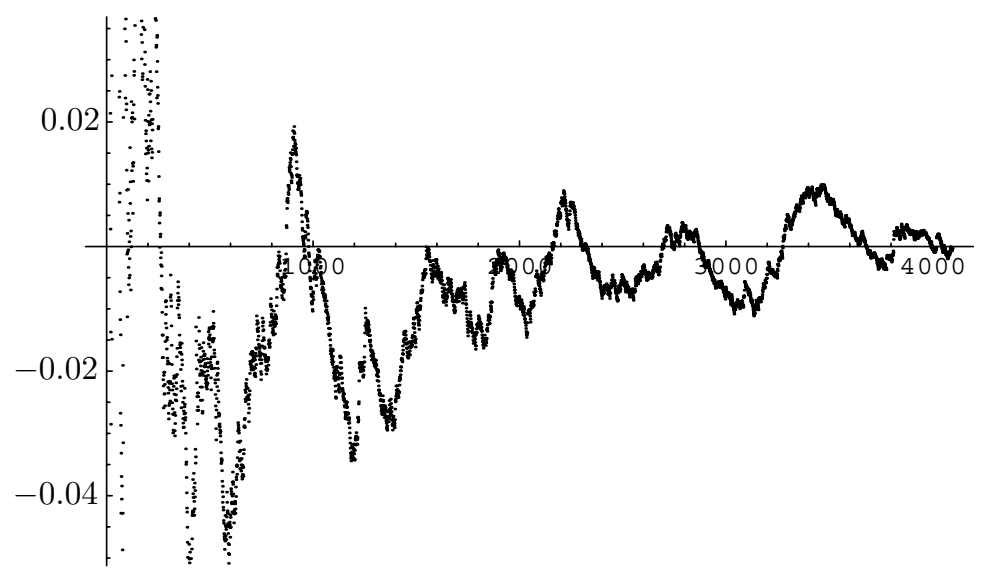

Figure 1. $x=\pi-3$

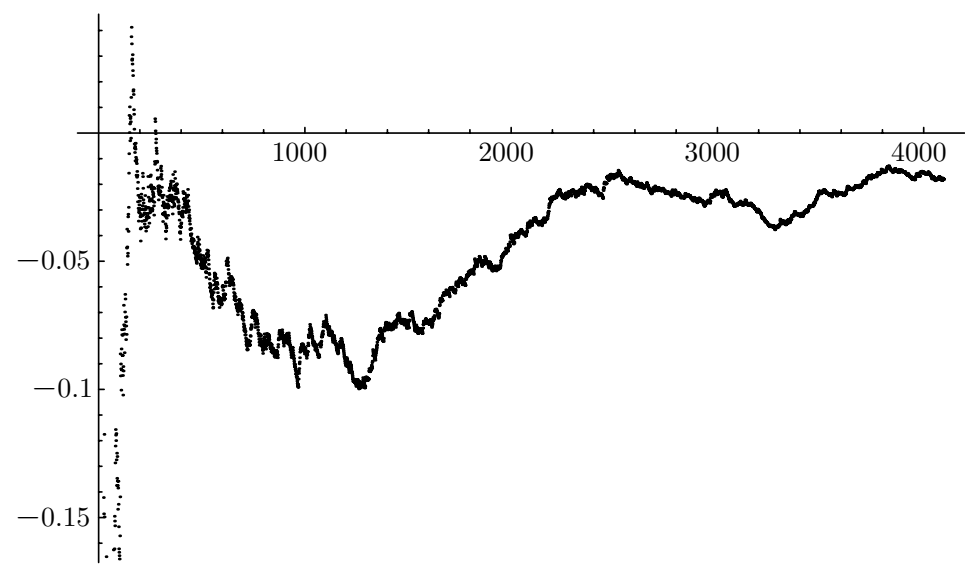

Figure 2. $x=K-2$

\section{The FunCtion $\phi$}

Let $\phi$ be the function defined on the interval $J=(0,1]$ as follows. For each $x$ in $J$ and for any positive integer $k$,

$$
\phi(x)=\log (k) \Longleftrightarrow v_{k+1}<x \leq v_{k} .
$$

In particular, for each positive integer $j, \phi\left(u_{j}\right)=\log \left(c_{j}\right)$. See Figure 3. Clearly,

$$
\begin{aligned}
\int_{J} \phi(x) \lambda(d x) & =\frac{1}{\log (2)} \sum_{k=1}^{\infty} \log (k)\left(\log \left(\frac{k+1}{k}\right)-\log \left(\frac{k+2}{k+1}\right)\right) \\
& =\frac{1}{\log (2)} \sum_{k=1}^{\infty} \log (k) \log \left(\frac{(k+1)^{2}}{k(k+2)}\right) \\
& =\log (K) .
\end{aligned}
$$




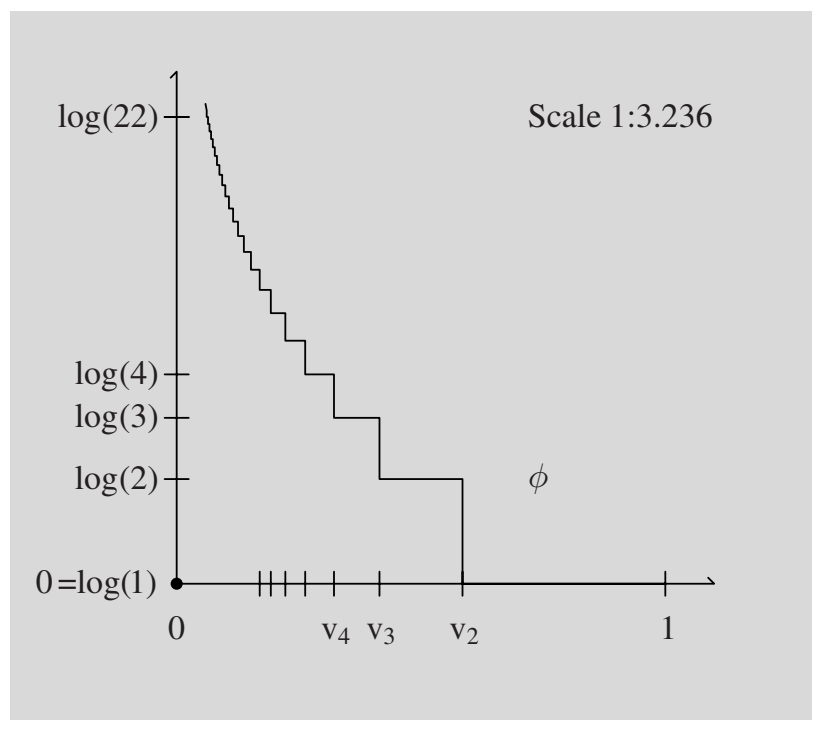

Figure 3. The function $\phi$

Now it is plain that $C$ is a Khinchin Sequence iff

$$
\lim _{n \rightarrow \infty} \frac{1}{n} \sum_{j=1}^{n} \phi\left(u_{j}\right)=\int_{J} \phi(x) \lambda(d x) .
$$

We proceed to prove relation (1).

\section{Integrating SEQUENCES}

Let $\psi$ be a real-valued Borel function defined on $J$ and integrable with respect to $\lambda$. Let us say that the sequence $U$ integrates $\psi$ iff

$$
\lim _{n \rightarrow \infty} \frac{1}{n} \sum_{j=1}^{n} \psi\left(u_{j}\right)=\int_{J} \psi(x) \lambda(d x) .
$$

To prove relation (1), we must prove that $U$ integrates $\phi$. Obviously, the functions integrated by $U$ comprise a linear space. By an elementary argument, one can show that, for each subinterval $I$ of $J, U$ integrates the characteristic function $\chi_{I}$ of $I$. One summarizes this property of $U$ by saying that $U$ is uniformly distributed in $J$. We will prove this property in an appendix to this paper. Presuming the property, let us prove that $U$ integrates $\phi$. To that end, we require only that:

(1) $\phi$ is nonnegative and decreasing;

(2) for each positive integer $p, U$ integrates the function

$$
\phi_{p}:=\chi_{\left[1 / 2^{p}, 1\right]} \phi .
$$

Let $n$ be any positive integer. Let $\alpha_{n}$ be the average of the values of $\phi$ at the first $n$ terms of $U$ :

$$
\alpha_{n}:=\frac{1}{n} \sum_{j=1}^{n} \phi\left(u_{j}\right) .
$$


Let

We must prove that

$$
\beta:=\int_{J} \phi(x) \lambda(d x)
$$

$$
\lim _{n \rightarrow \infty} \alpha_{n}=\beta .
$$

Let $p$ be any positive integer. Let $\phi_{p}$ be the function defined on $J$ by truncation of $\phi$, as follows:

That is,

$$
\phi_{p}:=\chi_{\left[1 / 2^{p}, 1\right]} \phi
$$

Obviously, for each $x$ in $J$,

$$
\phi_{p}(x):=\left\{\begin{array}{lll}
0 & \text { if } & 0<x<1 / 2^{p} \\
\phi(x) & \text { if } & 1 / 2^{p} \leq x \leq 1
\end{array}\right.
$$

$$
\phi_{1}(x) \leq \phi_{2}(x) \leq \cdots \leq \phi_{p}(x) \leq \cdots \uparrow \phi(x) .
$$

Let $\alpha_{n, p}$ be the corresponding average of the values of $\phi_{p}$ at the first $n$ terms of $U$ :

$$
\alpha_{n, p}:=\frac{1}{n} \sum_{j=1}^{n} \phi_{p}\left(u_{j}\right)
$$

Let

$$
\beta_{p}:=\int_{J} \phi_{p}(x) \lambda(d x)
$$

Clearly, $\phi_{p}$ is a linear combination of characteristic functions of subintervals of $J$. By our initial remarks, it is plain that $U$ integrates $\phi_{p}$ :

$$
\lim _{n \rightarrow \infty} \alpha_{p, n}=\beta_{p}
$$

Now let $\epsilon$ be any positive real number. By the Monotone Convergence Theorem, we may introduce a positive integer $p$ such that

$$
\beta-\epsilon<\beta_{p} \leq \beta,
$$

from which it follows that

$$
\int_{\left(0,1 / 2^{p}\right)} \phi(x) \lambda(d x)<\epsilon .
$$

By relation (2), we may introduce a positive integer $m$ such that, for every positive integer $n$, if $m \leq n$, then

$$
\beta_{p}-\epsilon<\alpha_{n, p}<\beta_{p}+\epsilon .
$$

We may as well arrange that $2^{p} \leq m$. Let $n$ be any positive integer for which $m \leq n$. Let $q$ be the positive integer for which $2^{q-1}-1<n \leq 2^{q}-1$. Clearly, $p<q$. One may say that the first $n$ terms of $U$ have run through the first $q-1$ "cycles" of $U$ and have at least begun (perhaps even finished) the $q$-th cycle. The smallest term of the $q$-th cycle is $1 / 2^{q}$. Hence, for each positive integer $j$, if $1 \leq j \leq n$, then $1 / 2^{q} \leq u_{j}$. Consequently,

$$
\alpha_{n, q}=\alpha_{n}
$$

Now let

be the terms among

$$
t_{1}, t_{2}, \ldots, t_{\ell} \quad\left(\ell=2^{q-p}-1\right)
$$

$$
u_{1}, u_{2}, \ldots, u_{r} \quad\left(r=2^{q}-1\right)
$$




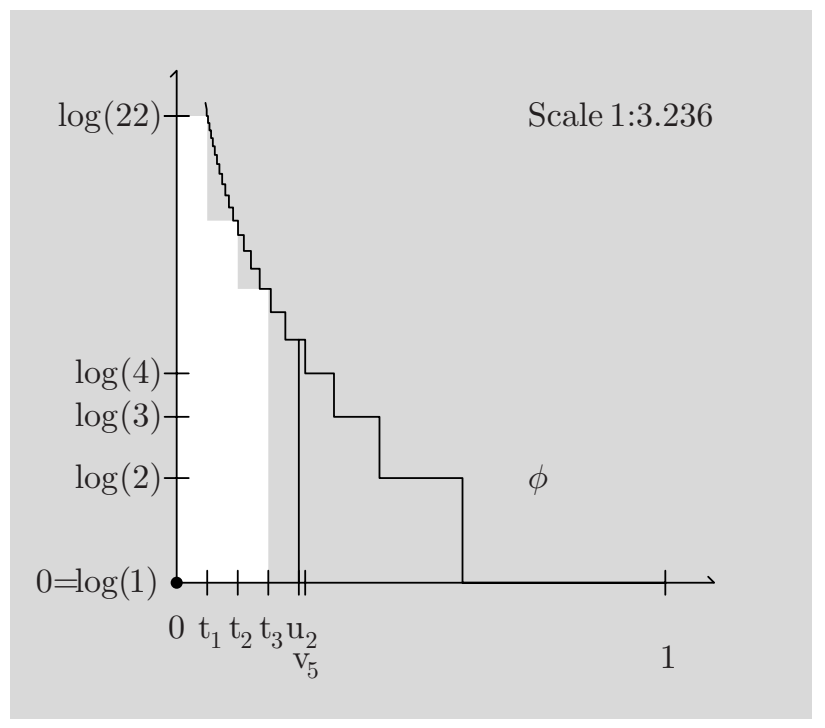

Figure 4. Comparison of areas

that are less than $1 / 2^{p}$. Figure $4, p=2, q=4$, and $\ell=3$. Since $\phi$ is nonnegative and decreasing, we find that

$$
\begin{array}{rlrl}
\alpha_{n, q}-\alpha_{n, p} & \leq \frac{1}{n} \sum_{j=1}^{\ell} \phi\left(t_{j}\right) & \\
& =\frac{2^{q}}{n} \frac{1}{2^{q}} \sum_{j=1}^{\ell} \phi\left(t_{j}\right) & \\
& \leq 4 \int_{\left(0,1 / 2^{p}\right)} \phi(x) \lambda(d x) & & \left(\text { since } 22^{q-1}<2(n+1)\right) \\
& <4 \epsilon \quad & & \text { (by relation }(4)) .
\end{array}
$$

Hence, by relations (3) and (5) and by the foregoing computation,

$$
\beta-2 \epsilon<\beta_{p}-\epsilon<\alpha_{n, p} \leq \alpha_{n, q}<\alpha_{n, p}+4 \epsilon<\beta_{p}+5 \epsilon \leq \beta+5 \epsilon
$$

so that, by relation (6), $\beta-2 \epsilon<\alpha_{n}<\beta+5 \epsilon$. Therefore,

$$
\lim _{n \rightarrow \infty} \frac{1}{n} \sum_{j=1}^{n} \phi\left(u_{j}\right)=\int_{J} \phi(x) \lambda(d v) .
$$

\section{Questions}

The number $x$ in $(0,1)$ of which $C$ is the continued fraction expansion is approximately equal to 0.46107049595671951935 . Of course, it is a Khinchin Number. Can one identify $x$ in "familiar" terms? 


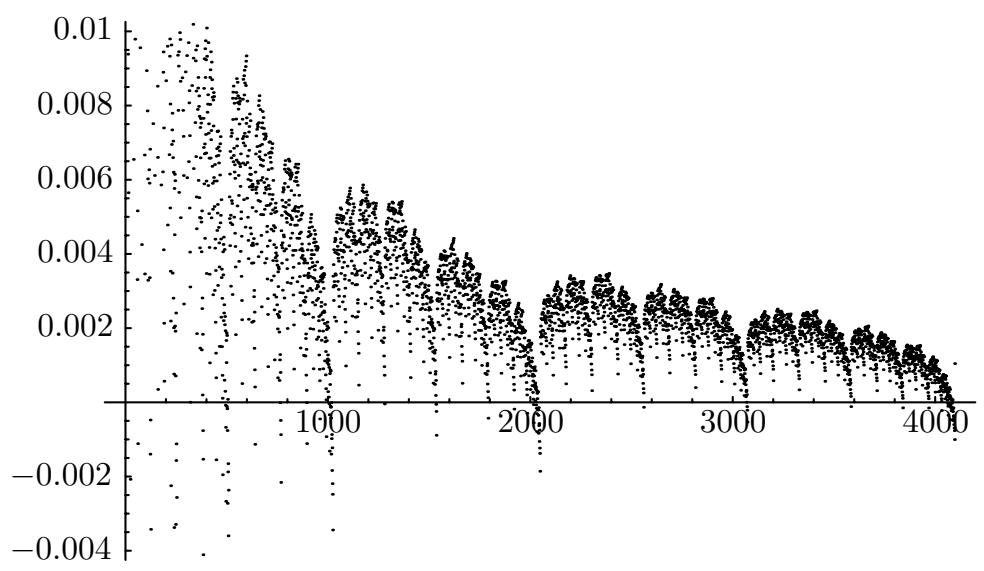

FiguRE 5. $N=4096$

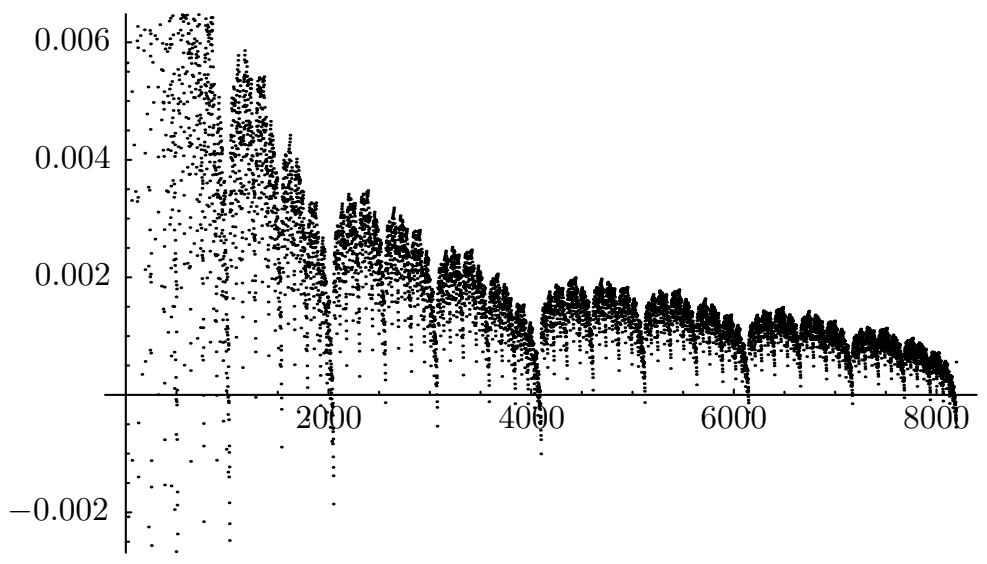

FiguRE $6 . \quad N=8192$

In Figures 5 and 6, we display list plots of the following data:

$$
\frac{1}{n} \sum_{j=1}^{n} \log \left(c_{j}\right)-\log (K) \quad(1 \leq n \leq N),
$$

where $N=4096$ and $N=8192$. Can one explain, in formally precise terms, the apparent self-similarity of the data?

\section{HARMONIC MEANS}

Let $r$ be any real number for which $r<1$ and $r \neq 0$. With reference to Section 4 , let us define the function $\phi_{r}$ on $J$ as follows. For each $x$ in $J$ and for any positive integer $k$,

$$
\phi_{r}(x)=k^{r} \Longleftrightarrow v_{k+1}<x \leq v_{k} .
$$

In particular, for each positive integer $j, \phi_{r}\left(u_{j}\right)=c_{j}^{r}$. If $r<0$, then $1-\phi_{r}$ is similar to $\phi$, in the sense that it meets the conditions (1) and (2) stated in Section 4. If 
$0<r<1$, then $\phi_{r}$ itself is similar to $\phi$. In either case, $U$ integrates $\phi_{r}$. Hence,

$$
\begin{aligned}
\lim _{n \rightarrow \infty} \frac{1}{n} \sum_{j=1}^{n} c_{j}^{r} & =\lim _{n \rightarrow \infty} \frac{1}{n} \sum_{j=1}^{n} \phi_{r}\left(u_{j}\right) \\
& =\int_{J} \phi_{r}(x) \lambda(d x) \\
& =\frac{1}{\log (2)} \sum_{k=1}^{\infty} k^{r} \log \left(\frac{(k+1)^{2}}{k(k+2)}\right) .
\end{aligned}
$$

One defines the Khinchin Constant $K_{r}$ by the following relation:

$$
K_{r}^{r}=\frac{1}{\log (2)} \sum_{k=1}^{\infty} k^{r} \log \left(\frac{(k+1)^{2}}{k(k+2)}\right) .
$$

We infer that the $r$-th harmonic means of $C$ converge to $K_{r}$ :

$$
\lim _{n \rightarrow \infty}\left(\frac{1}{n} \sum_{j=1}^{n} c_{j}^{r}\right)^{1 / r}=K_{r}
$$

\section{APPENDiX}

The van der Corput Sequence $U$ falls into cycles:

$$
U: \quad \frac{1}{2}, \quad \frac{1}{4}, \frac{3}{4}, \frac{1}{8}, \frac{5}{8}, \frac{3}{8}, \frac{7}{8}, \frac{1}{16}, \frac{9}{16}, \frac{5}{16}, \frac{13}{16}, \frac{3}{16}, \frac{11}{16}, \frac{7}{16}, \frac{15}{16}, \ldots, u_{j}, \ldots
$$

For each positive integer $p$, the first term of the $p$-th cycle is $1 / 2^{p}$ and the length of the $p$-th cycle is $2^{p-1}$. The sum of the lengths of the first $p$ cycles is $2^{p}-1$. Moreover,

$$
u_{2^{p}+j}=\frac{1}{2^{p+1}}+u_{j} \quad\left(0<j<2^{p}\right) .
$$

By these observations, it is plain that, for any dyadic interval $I$ of the form

$$
I=\left[j / 2^{p},(j+1) / 2^{p}\right) \quad\left(p \in \mathbb{Z}^{+}, 0<j<2^{p}\right),
$$

the sequence $U$ visits $I$ precisely once in the course of its first $p$ cycles. Thereafter, it visits $I$ periodically with period $2^{p}$. Hence, for any positive integer $n$, if $2^{p} \leq n$, then

$$
\frac{m}{n} \leq \frac{1}{n} \sum_{j=1}^{n} \chi_{I}\left(u_{j}\right) \leq \frac{m+1}{n},
$$

where $m$ is the positive integer for which

$$
m 2^{p}-1<n \leq(m+1) 2^{p}-1 .
$$

Consequently,

$$
\lim _{n \rightarrow \infty} \frac{1}{n} \sum_{j=1}^{n} \chi_{I}\left(u_{j}\right)=\frac{1}{2^{p}}=\lambda(I),
$$

which is to say that $U$ integrates $\chi_{I}$. 
In turn, for any subinterval $I$ of the interval $(0,1)$ and for any positive real number $\epsilon$, we may introduce finite disjoint unions $I^{\prime}$ and $I^{\prime \prime}$ of dyadic intervals of the foregoing form such that $I^{\prime} \subseteq I \subseteq I^{\prime \prime}$ and $\lambda\left(I^{\prime \prime} \backslash I^{\prime}\right)<\epsilon$. Hence,

$$
\begin{aligned}
\limsup _{n \rightarrow \infty} \frac{1}{n} \sum_{j=1}^{n} \chi_{I}\left(u_{j}\right) & \leq \lim _{j \rightarrow \infty} \frac{1}{n} \sum_{j=1}^{n} \chi_{I^{\prime \prime}}\left(u_{j}\right) \\
& =\lambda\left(I^{\prime \prime}\right) \\
& <\lambda\left(I^{\prime}\right)+\epsilon \\
& =\lim _{n \rightarrow \infty} \frac{1}{n} \sum_{j=1}^{n} \chi_{I^{\prime}}\left(u_{j}\right)+\epsilon \\
& \leq \liminf _{n \rightarrow \infty} \frac{1}{n} \sum_{j=1}^{n} \chi_{I}\left(u_{j}\right)+\epsilon .
\end{aligned}
$$

Consequently,

$$
\lim _{n \rightarrow \infty} \frac{1}{n} \sum_{j=1}^{n} \chi_{I}\left(u_{j}\right)=\lambda(I),
$$

which is to say that $U$ integrates $\chi_{I}$.

\section{REFERENCES}

[1] D. H. Bailey, J. M. Borwein, R. C. Crandall, On the Khinchin Constant, Math. Comp. 66 (1997), 417-431. MR1377659 (97c:11119)

[2] J. G. van der Corput, Verteilungsfunktionen, Proc. Ned. Akad. v. Wet. 38 (1935), 813-821.

[3] D. H. Lehmer, Note on an Absolute Constant of Khinchin, Amer. Math. Monthly, 46 (1939), 148-152. MR1524526

[4] C. Ryll-Nardzewski, On the Ergodic Theorems (I, II), Studia Math. 12 (1951), 65-79. MR0046582 (13:757a); MR0046583 (13:757b)

[5] P. Walters, An Introduction to Ergodic Theory, Springer-Verlag, New York, 1982. MR648108 (84e:28017)

Department of Mathematics, Reed College, Portland, Oregon 97202

E-mail address: wieting@reed.edu 